\title{
Détermination de courbes elliptiques pour la conjecture de Szpiro
}

\author{
par \\ Abderrahmane Nitaj (Saarbrücken)
}

1. Introduction. En 1981, Szpiro [16] a proposé une conjecture reliant le discriminant d'une courbe elliptique et son conducteur. Une version effective de cette conjecture est la suivante [17].

Conjecture (Szpiro). Pour tout $\varepsilon>0$, il existe une constante $C_{\varepsilon}$ telle que si $E / \mathbb{Q}$ est une courbe elliptique de discriminant minimal $\Delta$ et de conducteur $N$, alors

$$
|\Delta| \leq C_{\varepsilon} N^{6+\varepsilon} \text {. }
$$

Cette conjecture est très utile pour l'étude des équations diophantiennes exponentielles, et son analogue pour les corps de fonctions est démontré (voir [7] et [17]). Elle implique que le rapport

$$
\sigma=\sigma(E)=\frac{\log |\Delta|}{\log N}
$$

appelé rapport de Szpiro, est borné. On peut montrer en fait qu'il existe une infinité de courbes elliptiques pour lesquelles $\sigma \geq 6$ (voir [13]). La valeur asymptotique conjecturale de $\sigma$ est donc 6 . D'autre part, Fouvry et al. [6] ont montré que pour toute constante $K>1$, la proportion des courbes elliptiques $E$, semi-stables, avec $\sigma(E)>K$ est nulle et que le rapport de Szpiro est "presque partout" voisin de 1.

Le but de cet article est de décrire des méthodes qui permettent de déterminer des courbes elliptiques ayant un rapport de Szpiro (1) assez grand. Par exemple, la courbe elliptique définie par l'équation

$y^{2}+x y=x^{3}+x^{2}+349410011109107572 x-775428774618307505842556592$

1991 Mathematics Subject Classification: 11D41, 11 Y99.

Key words and phrases: courbe elliptique, isogénie, conjecture de Szpiro.

Research supported by the TMR programme of the European Community under contract ERBFMBICT960848. 
admet pour rapport de Szpiro

$$
\sigma=\frac{\log \left(2^{26} \cdot 3^{52} \cdot 5 \cdot 11^{8} \cdot 13 \cdot 19^{6} \cdot 31^{4}\right)}{\log (2 \cdot 3 \cdot 5 \cdot 11 \cdot 13 \cdot 19 \cdot 31)} \simeq 8.811944
$$

Cet exemple provient en fait de la famille des courbes elliptiques d'équation $y^{2}=x\left(x^{2}+2(s+t) x+(s-t)^{2}\right)$ et de discriminant $\Delta=2^{8} s t(s-t)^{4}$ (voir partie 4), avec ici $s=2^{30} \cdot 5, t=-13 \cdot 19^{6}$ et $s-t=3^{13} \cdot 11^{2} \cdot 31$. Ceci met en évidence un lien entre la conjecture de Szpiro et les "bons exemples" de la conjecture $a b c$ (voir [11]). Ces exemples ont été utilisés par ailleurs par de Weger [20] pour construire des courbes elliptiques ayant un grand groupe de Tate-Shafarevich.

Plus généralement, soit $E(s, t)$ une courbe elliptique définie sur $\mathbb{Q}(s, t)$ par l'équation de Weierstrass :

$E(s, t): \quad y^{2}+a_{1}(s, t) x y+a_{3}(s, t) y=x^{3}+a_{2}(s, t) x^{2}+a_{4}(s, t) x+a_{6}(s, t)$, avec $a_{1}, a_{2}, a_{3}, a_{4}, a_{6} \in \mathbb{Z}[s, t]$. Soit $P=(x, y)$ un point rationnel de $E(s, t)$, d'ordre $m \in\{2,3, \ldots, 8\}$. Le choix de telles familles de courbes elliptiques est basé sur trois remarques, concernant le discriminant $\Delta(s, t)$ de $E(s, t)$ :

(i) Si $m \geq 3$, le théorème de Nagell-Lutz (voir [8] ou [14]) implique que $4 \Delta(s, t)$ est divisible par $\left(2 y+a_{1} x+a_{3}\right)^{2}$.

(ii) Le discriminant s'écrit sous la forme $\Delta(s, t)=f(s, t)^{m} g(s, t)$, avec $f, g \in \mathbb{Z}[s, t]$.

(iii) Si on désigne par $N_{0}(s, t)$ le conducteur de $E(s, t)$, alors il y a égalité dans la relation de Kodaira :

$$
\operatorname{deg} \Delta(s, t) \leq 6 \operatorname{deg} N_{0}(s, t)-12 .
$$

Cette inégalité est l'équivalent de la conjecture de Szpiro pour les corps de fonctions de genre et caractéristique nuls (voir [7] et [17] pour d'autres versions). Les remarques (ii) et (iii) sont aussi vérifiées par les différentes isogènes de $E(s, t)$.

Dans ce travail, la recherche de courbes elliptiques ayant un grand rapport de Szpiro se résume de la façon suivante. On commence par exhiber une courbe elliptique dépendant de deux paramètres $s$ et $t$, pour laquelle le point

$$
P_{0}=(0,0)
$$

est un point de torsion d'ordre $m \in\{2,3, \ldots, 8\}$. On détermine ensuite toutes ses isogènes et on cherche, en résolvant certaines équations diophantiennes, des valeurs particulières de $s$ et $t$ qui rendent le rapport (1) assez grand. Ces résultats sont listés dans des tables contenant les dix grandes valeurs du rapport (1) ainsi obtenues pour chaque valeur de $m$. On calcule ensuite le rapport de Szpiro de certaines tordues quadratiques des courbes 
elliptiques dans chaque table, et on liste les six meilleurs exemples. Dans toutes les tables, $T(E)$ désigne le sous-groupe des points de torsion de $E$ :

$$
T(E)=E(\mathbb{Q})_{\text {tors }} .
$$

Tous les calculs ont été effectués en utilisant le système SIMATH [15]. On peut cependant vérifier les résultats à l'aide d'autres systèmes de calcul comme PARI [2] ou APECS [4].

Le plan du présent article est le suivant. On rappelle dans la partie 2 quelques notions utiles concernant les courbes elliptiques, la partie 3 décrit les différentes méthodes employées pour déterminer des courbes elliptiques ayant un grand rapport de Szpiro, et les parties 4 à 10 sont consacrées à la recherche de telles courbes. Enfin, dans la partie 11, on donne brièvement la raison pour laquelle on s'est limité aux courbes elliptiques ayant un point de torsion d'ordre $m \in\{2,3, \ldots, 8\}$.

Je tiens à remercier le Professeur H. G. Zimmer qui m'a indiqué l'article de T. Nagell [10], un des travaux pionniers dans le domaine de la recherche des points de torsion des courbes elliptiques.

2. Points de torsion et isogénies. Soit $E$ une courbe elliptique définie par l'équation de Weierstrass :

$$
E: \quad y^{2}+a_{1} x y+a_{3} y=x^{3}+a_{2} x^{2}+a_{4} x+a_{6},
$$

avec $a_{1}, a_{2}, a_{3}, a_{4}, a_{6} \in \mathbb{Z}$. On note $O$ le point à l'infini de $E$. Les quantités habituelles $b_{2}, b_{4}, b_{6}, b_{8}, c_{4}$ et $c_{6}$, liées à $E$, sont données par (voir [14]) :

$$
\begin{gathered}
b_{2}=a_{1}^{2}+4 a_{2}, \quad b_{4}=a_{1} a_{3}+2 a_{4}, \quad b_{6}=a_{3}^{2}+4 a_{6}, \quad b_{8}=\left(b_{2} b_{6}-b_{4}^{2}\right) / 4, \\
c_{4}=b_{2}^{2}-24 b_{4}, \quad c_{6}=-b_{2}^{3}+36 b_{2} b_{4}-216 b_{6} .
\end{gathered}
$$

Le discriminant de $E$ est alors $\Delta=\left(c_{4}^{3}-c_{6}^{2}\right) / 1728$. Si $\Delta$ est minimal, le conducteur $N$ de $E$ est (voir [14], p. 361)

$$
N=\prod_{p \mid \Delta} p^{n_{p}}, \quad p \text { premier }
$$

avec

$$
n_{p}= \begin{cases}1 & \text { si } E \text { a une réduction multiplicative en } p \\ 2 & \text { si } E \text { a une réduction additive en } p \geq 5, \\ 2+\varepsilon_{p} & \text { si } E \text { a une réduction additive en } p=2,3\left(0 \leq \varepsilon_{p} \leq 6\right) .\end{cases}
$$

Ainsi, si $E$ admet en tout nombre premier une bonne réduction ou une réduction multiplicative ( $E$ est dite semi-stable), son conducteur est le produit des facteurs premiers de son discriminant minimal.

On considère le polynôme $\Psi_{n}(x, y)$ défini pour tout $n \geq 0$ à partir des formules de récurrence (voir [1], [18]) 


$$
\begin{aligned}
\Psi_{0}= & 0, \quad \Psi_{1}=1, \quad \Psi_{2}=2 y+a_{1} x+a_{3}, \\
\Psi_{3}= & 3 x^{4}+b_{2} x^{3}+3 b_{4} x^{2}+3 b_{6} x+b_{8}, \\
\Psi_{4}= & \Psi_{2}\left(2 x^{6}+b_{2} x^{5}+5 b_{4} x^{4}+10 b_{6} x^{3}+10 b_{8} x^{2}\right. \\
& \left.+\left(b_{2} b_{8}-b_{4} b_{6}\right) x+b_{4} b_{8}-b_{6}^{2}\right),
\end{aligned}
$$

et pour $m \geq 2$,

$$
\begin{aligned}
& \Psi_{2 m+1}=\Psi_{m+2} \Psi_{m}^{3}-\Psi_{m-1} \Psi_{m+1}^{3}, \\
& \Psi_{2 m}=\Psi_{m}\left(\Psi_{m+2} \Psi_{m-1}^{2}-\Psi_{m-2} \Psi_{m+1}^{2}\right) / \Psi_{2}
\end{aligned}
$$

On a en particulier l'égalité

$$
\Psi_{2}^{2}=4 x^{3}+b_{2} x^{2}+2 b_{4} x+b_{6} .
$$

Soit $P=(x, y) \neq O$ un point de $E(\mathbb{Q})$. Alors $P$ est un point de torsion d'ordre $m \geq 2$ si, et seulement si, $\Psi_{m}(x, y)=0$. De plus, si $P=(x, y)$ est un point de 2-torsion, alors $y=-\left(a_{1} x+a_{3}\right) / 2$.

Si une courbe elliptique $E$ admet un sous-groupe fini $G$, alors il existe une unique courbe elliptique $E / G$ et une isogénie $\phi$ de $E$ dans $E / G$, ayant $G$ pour noyau (voir [14], p. 78). L'équation de $E / G$ et l'expression de $\phi$ peuvent être déterminées par les formules de Vélu [19], de la façon suivante. Soit $F_{2}$ le sous-groupe des points d'ordre 2 de $G-\{O\}$. On peut écrire $G-\{O\}=F_{2} \cup R \cup(-R)$, avec $R \cap(-R)=\emptyset$. Soit $S=R \cup F_{2}$. Pour $Q=\left(x_{Q}, y_{Q}\right) \in S$, on pose

$$
\begin{aligned}
g_{Q}^{x} & =3 x_{Q}^{2}+2 a_{2} x_{Q}+a_{4}-a_{1} y_{Q}, \\
g_{Q}^{y} & =-2 y_{Q}-a_{1} x_{Q}-a_{3}, \\
u_{Q} & =4 x_{Q}^{3}+b_{2} x_{Q}^{2}+2 b_{4} x_{Q}+b_{6}, \\
t_{Q} & = \begin{cases}x_{Q}^{2}+2 a_{2} x_{Q}+a_{4}-a_{1} y_{Q} & \text { si } Q \in F_{2}, \\
6 x_{Q}^{2}+b_{2} x_{Q}+b_{4} & \text { si } Q \in R,\end{cases} \\
U_{Q} & =\frac{t_{Q}}{x-x_{Q}}+\frac{u_{Q}}{\left(x-x_{Q}\right)^{2}}, \\
V_{Q} & =\frac{2 y+a_{1} x+a_{3}}{\left(x-x_{Q}\right)^{3}}+t_{Q} \frac{a_{1}\left(x-x_{Q}\right)+y-y_{Q}}{\left(x-x_{Q}\right)^{2}}+\frac{a_{1} u_{Q}-g_{Q}^{x} g_{Q}^{y}}{\left(x-x_{Q}\right)^{2}},
\end{aligned}
$$

et enfin

$$
t=\sum_{Q \in S} t_{Q}, \quad w=\sum_{Q \in S}\left(u_{Q}+x_{Q} t_{Q}\right) .
$$

ThÉorème 1 (Vélu). Une isogénie $\phi: E \rightarrow E / G$, qui envoie $(x, y)$ sur $(X, Y)$ est donnée par

$$
X=x+\sum_{Q \in S} U_{Q}, \quad Y=y-\sum_{Q \in S} V_{Q} .
$$


L'équation de $E / G$ est donnée par l'équation

$$
Y^{2}+a_{1} X Y+a_{3} Y=X^{3}+a_{2} X^{2}+\left(a_{4}-5 t\right) X+a_{6}-b_{2} t-7 w .
$$

\section{Méthodes utilisées}

3.1. Recherche directe. Soit $E$ une courbe elliptique ayant $P_{0}$ pour point de torsion d'ordre $m \geq 2$. D'après le théorème de Mazur (voir [8]), $m \in$ $\{2,3, \ldots, 10,12\}$. D'autre part, ces courbes dépendent de plusieurs paramètres (voir [8], [9] ou [10]), mais on peut les ramener à deux paramètres $s$ et $t$ :

(3) $E(s, t): y^{2}+a_{1}(s, t) x y+a_{3}(s, t) y=x^{3}+a_{2}(s, t) x^{2}+a_{4}(s, t) x$.

Pour $m \geq 4$, la courbe de Tate (voir [8]) définie par

$$
E(A, B, C): \quad y^{2}+(C-A) x y-B C^{2} y=x^{3}-B C x^{2},
$$

admet $P_{0}$ comme point de torsion d'ordre $m$, suivant les valeurs de $A, B$ et $C$. Dans tous les cas, cette courbe se ramène à la forme (3). A part les cas $m=2$ et $m=3$, le discriminant de (3) se factorise sous la forme

$$
\Delta=\Delta(s, t)=K s^{e} t^{f} \prod_{i=1}^{g} F_{i}^{n_{i}}(s, t),
$$

où $K$ est une constante, et pour $1 \leq i \leq g, F_{i}$ est une forme binaire irréductible sur $\mathbb{Q}$, de degré $n \geq 1$. Les grandes valeurs du rapport de Szpiro correspondent à des discriminants grands par rapport aux conducteurs.

Une première méthode consiste à déterminer des entiers $s, t$ et $z$, vérifiant l'équation

$$
F(s, t)=M z=z \prod_{j=1}^{k} p_{j}^{e_{j}},
$$

où $F=F_{i}$ est l'une des formes binaires composant $\Delta, p_{1}, \ldots, p_{k}$ des nombres premiers fixés, $e_{j} \geq 1$, et $|z|$ est assez petit par rapport à $M$. Si le degré de $F$ est $n=1$, on applique l'algorithme d'Euclide. Supposons donc que $n \geq 2$. On pose

$$
\begin{aligned}
& S_{1}=\{\theta: \theta \in \mathbb{C}, F(\theta, 1)=0\}, \\
& S_{2}=\{T: T \in \mathbb{Z}, 1 \leq T<M, F(T, 1) \equiv 0(\bmod M)\} .
\end{aligned}
$$

La détermination de $S_{2}$ se fait à l'aide du lemme de Hensel, combiné avec la méthode de Shanks si $n=2$ ou la méthode de Berlekamp si $n \geq 3$ et à l'aide du théorème chinois (voir [3]). Soit $(s, t, z)$ une solution de (5), vérifiant $(t, M)=1$. Soit $\theta \in S_{1}$ vérifiant

$$
|s-t \theta| \leq \min _{\theta_{i} \in S_{1}}\left|s-t \theta_{i}\right|
$$


On désigne par $\Re(\theta)$ la partie réelle de $\theta$ et par $\Im(\theta)$ sa partie imaginaire. Soit $\alpha \geq 0$. Si $\Im(\theta) \neq 0$, on pose

$$
V_{0}=V_{0}(\theta)=\left(\frac{2^{n-1} M^{1+\alpha}}{\left|F^{\prime}(\theta, 1) \Im(\theta)\right|}\right)^{1 / n} .
$$

Si $n \geq 3$, on pose

$$
V_{1}=V_{1}(\theta)=\left(\frac{2^{n} M^{\alpha}}{\left|F^{\prime}(\theta, 1)\right|}\right)^{1 /(n-2)} .
$$

Lemme 2. Soient $\alpha \geq 0$ et $(s, t, z)$ une solution de (5) vérifiant $(t, M)=1$ et $|z| \leq M^{\alpha}$. Alors il existe une racine $\theta \in S_{1}$ et un entier $T \in S_{2}$ tels que :

(a) si $n=2$ et $\left|F^{\prime}(\theta, 1)\right| \geq 4 M^{\alpha}$ ou si $n \geq 3$ et $|t| \geq V_{1}$, alors il existe un entier $u$ tel que $s=T t-M u$ et $u / t$ est une réduite de la fraction continue de $(T-\Re(\theta)) / M$,

(b) si de plus $\Im(\theta) \neq 0$, alors $|t| \leq V_{0}$.

Preuve. (a) D'après (6), pour tout $\theta_{i} \in S_{1}$ on a

ce qui donne

$$
\left|\frac{s}{t}-\theta_{i}\right| \geq \frac{1}{2}\left|\frac{s}{t}-\theta_{i}\right|+\frac{1}{2}\left|\frac{s}{t}-\theta\right| \geq \frac{1}{2}\left|\theta-\theta_{i}\right|,
$$

$$
\prod_{\theta_{i} \neq \theta}\left|\frac{s}{t}-\theta_{i}\right| \geq \frac{1}{2^{n-1}} \prod_{\theta_{i} \neq \theta}\left|\theta-\theta_{i}\right|=\frac{\left|F^{\prime}(\theta, 1)\right|}{2^{n-1}\left|a_{0}\right|},
$$

où $a_{0}$ est le coefficient de $s^{n}$ dans $F(s, t)$. D'autre part, on a

$$
|F(s, t)|=\left|a_{0}\right| \cdot|t|^{n}\left|\frac{s}{t}-\theta\right| \prod_{\theta_{i} \neq \theta}\left|\frac{s}{t}-\theta_{i}\right|,
$$

d'où

$$
\left|\frac{s}{t}-\theta\right| \leq \frac{2^{n-1}|F(s, t)|}{\left|F^{\prime}(\theta, 1)\right| \cdot|t|^{n}} \leq \frac{2^{n-1} M^{1+\alpha}}{\left|F^{\prime}(\theta, 1)\right| \cdot|t|^{n}} .
$$

Soit $T \equiv s t^{-1}(\bmod M)$ avec $1 \leq T<M$, alors $T \in S_{2}$. Soit $u$ l'entier vérifiant $s=T t-M u$. L'inégalité ci-dessus devient

$$
\left|\frac{u}{t}-\frac{T-\theta}{M}\right| \leq \frac{2^{n-1} M^{\alpha}}{\left|F^{\prime}(\theta, 1)\right| \cdot|t|^{n}} .
$$

Si $n=2$, cette inégalité donne

$$
\left|\frac{u}{t}-\frac{T-\Re(\theta)}{M}\right| \leq \frac{2 M^{\alpha}}{\left|F^{\prime}(\theta, 1)\right| t^{2}},
$$

et donc, si $\left|F^{\prime}(\theta, 1)\right| \geq 4 M^{\alpha}$, alors $u / t$ est une réduite de la fraction continue de $(T-\Re(\theta)) / M$. 
Si $n \geq 3$, alors (7) donne

$$
\left|\frac{u}{t}-\frac{T-\Re(\theta)}{M}\right| \leq\left(\frac{V_{1}}{|t|}\right)^{n-2} \frac{1}{2 t^{2}}
$$

et si $|t| \geq V_{1}, u / t$ est une réduite de la fraction continue de $(T-\Re(\theta)) / M$.

(b) Si de plus $\Im(\theta) \neq 0$, alors (7) donne

$$
|\Im(\theta)| \leq\left(\frac{V_{0}}{|t|}\right)^{n}|\Im(\theta)|
$$

et donc $|t| \leq V_{0}$.

Remarque 1. Dans la pratique, ce lemme sera utilisé pour déterminer des entiers $s$ et $t$ vérifiant l'équation (5), à partir des premières réduites de $(T-\Re(\theta)) / M$, où $\theta \in S_{1}$ et $T \in S_{2}$. La restriction sur $z$ sera donc ignorée.

Les factorisations des discriminants des courbes elliptiques étudiées dans la suite nous amènent à traiter les équations suivantes :

$$
\begin{gathered}
a X^{n}-b Y^{n}=M z, \quad n \geq 1, \\
X^{2}+11 X Y-Y^{2}=M z, \\
X^{3}-8 X^{2} Y+5 X Y^{2}+Y^{3}=M z, \\
8 X^{2}-8 X Y+Y^{2}=M z .
\end{gathered}
$$

Les valeurs de $n, a, b$ et $M$ seront fixées à l'occasion de chaque appel à l'une de ces équations. L'équation (8) a déjà servi par ailleurs à chercher des exemples optimaux pour la conjecture $a b c$ (voir [11]).

3.2. Utilisation des tordues quadratiques. Soit $d$ un entier sans facteurs carrés. La tordue quadratique $E^{(d)}$ d'une courbe elliptique $E$ donnée par (2) a pour équation

$$
E^{(d)}: \quad y^{2}+a_{1}^{(d)} x y+a_{3}^{(d)} y=x^{3}+a_{2}^{(d)} x^{2}+a_{4}^{(d)} x+a_{6}^{(d)},
$$

avec

$$
\begin{aligned}
& a_{1}^{(d)}=a_{1}, \quad a_{2}^{(d)}=a_{2} d+\frac{a_{1}^{2}(d-1)}{4}, \quad a_{3}^{(d)}=a_{3}, \\
& a_{4}^{(d)}=a_{4} d^{2}+\frac{a_{1} a_{3}\left(d^{2}-1\right)}{2}, \quad a_{6}^{(d)}=a_{6} d^{3}+\frac{a_{3}^{2}\left(d^{3}-1\right)}{4} .
\end{aligned}
$$

Ses covariants $\Delta^{(d)}$ et $c_{4}^{(d)}$, non nécessairement minimaux, sont liés à ceux de $E$ par les relations

$$
\Delta^{(d)}=d^{6} \Delta, \quad c_{4}^{(d)}=d^{2} c_{4} .
$$

Le conducteur de $E^{(d)}$ est composé des facteurs premiers de $4 N d$, et, dans un cas particulier, on a la proposition suivante, relative à son rapport de Szpiro $\sigma^{(d)}$. 
Proposition 3. Soit E une courbe elliptique semi-stable de discriminant minimal $\Delta$, de conducteur $N$ et de rapport de Szpiro $\sigma$. Soit d un facteur de $\pm N$. Le rapport de Szpiro $\sigma^{(d)}$ de la tordue quadratique $E^{(d)}$ de $E$ vérifie

$$
\frac{\sigma \log N+6 \log |d|}{\log (16|d| N)} \leq \sigma^{(d)} \leq \frac{\sigma \log N+6 \log |d|}{\log (|d| N)} .
$$

De plus, si $6 \log |d| / \log (16|d|)>\sigma$, alors $\sigma^{(d)}>\sigma$ et si $\sigma>6$, alors $\sigma^{(d)}<\sigma$.

Pre uve. Puisque $E$ est semi-stable, son conducteur $N$ est sans facteurs carrés. Le conducteur de $E^{(d)}$ est (voir [5]) $N^{(d)}=N D^{2} /\left(N, D^{2}\right)$, où $D$ est le discriminant de $\mathbb{Q}(\sqrt{d})$, donné par

$$
D= \begin{cases}d & \text { si } d \equiv 1(\bmod 4) \\ 4 d & \text { si } d \equiv 2,3(\bmod 4) .\end{cases}
$$

Alors, pour tout nombre premier $p \geq 2$, on a

$$
\operatorname{ord}_{p}\left(N^{(d)}\right)=\max \left(\operatorname{ord}_{p}(N), 2 \operatorname{ord}_{p}(D)\right)
$$

En posant

$$
\nu=\max \left(\operatorname{ord}_{2}(N), 2 \operatorname{ord}_{2}(D)\right)-\operatorname{ord}_{2}(N)-\operatorname{ord}_{2}(d),
$$

et comme $d$ divise $N$, on obtient $\nu \in\{0,3,4\}$ et $N^{(d)}=2^{\nu} N|d|$. Alors $\Delta^{(d)}$ est minimal et

$$
\sigma^{(d)}=\frac{\log \Delta^{(d)}}{\log N^{(d)}}=\frac{\sigma \log N+6 \log |d|}{\log \left(2^{\nu}|d| N\right)}
$$

ce qui donne les deux inégalités de la proposition. On obtient de même

$$
\sigma^{(d)}-\sigma=\frac{6 \log |d|-\sigma \log \left(2^{\nu}|d|\right)}{\log \left(2^{\nu}|d| N\right)} .
$$

Si $|d|=1$, alors $d=-1$ et $\nu \geq 3$. Dans tous les cas, le signe de $\sigma^{(d)}-\sigma$ est le même que celui de la différence $6 \log |d| / \log \left(2^{\nu}|d|\right)-\sigma$, d'où la conclusion de la proposition en remarquant que $6 \log |d| / \log \left(2^{\nu}|d|\right) \leq 6$.

REMARQUe 2. Les courbes elliptiques qui seront déterminées par la méthode directe auront toutes un rapport $\sigma>7.2$. Alors, d'après la conclusion de la proposition 3, on a $\sigma^{(d)}<\sigma$. Ainsi, cette méthode ne permet pas d'améliorer les valeurs du rapport (1). Cependant, elle donne de nouvelles courbes elliptiques avec un rapport $\sigma^{(d)}$ assez grand d'une part, et permet d'instaurer une hiérarchie entre les tordues quadratiques des courbes d'une même famille d'autre part.

4. Points de 2-torsion. Pour avoir $2 P_{0}=O$ sur la courbe elliptique (3), on doit avoir $\Psi_{2}(0,0)=0$, donc $a_{3}=0$ et $a_{4} \neq 0$. Le changement de variables 
$(x, y)=\left(4 x, 8 y+4 a_{1} x\right)$ donne la nouvelle équation

$$
E: y^{2}=x\left(x^{2}+A x+8 B\right),
$$

avec $A=4 a_{2}+a_{1}^{2}, B=2 a_{4}$. Si $P=(x, y)$ est d'ordre 2, alors $\Psi_{2}(x, y)=0$, i.e. $x\left(x^{2}+A x+8 B\right)=0$.

4.1. Supposons que le polynôme $x^{2}+A x+8 B$ est irréductible sur $\mathbb{Q}$. On pose $s=A, t=8 B$ et

$$
E_{1}: \quad y^{2}=x\left(x^{2}+s x+t\right) .
$$

Le discriminant de $E_{1}$ est alors

$$
\Delta_{1}=2^{4} t^{2}\left(s^{2}-4 t\right)
$$

En divisant $E_{1}$ par le sous-groupe $\left\{O, P_{0}\right\}$, et en effectuant le changement $(x, y)=(x+s, y)$, on obtient la courbe

$$
E_{2}: \quad y^{2}=x\left(x^{2}-2 s x+s^{2}-4 t\right)
$$

avec pour discriminant

$$
\Delta_{2}=2^{8} t\left(s^{2}-4 t\right)^{2} .
$$

Si on pose $s=2(u+v)$ et $t=(u-v)^{2}$, alors $E_{1}(s, t)=E_{4}(u, v)$ et $E_{2}(s, t)=$ $E_{3}(4 u, 4 v)$ (voir partie 4.2 ci-dessous), ce qui permet d'utiliser la table 3. Pour avoir des courbes qui ne sont pas de cette famille, on peut considérer l'équation $X^{2}-b Y^{2}=M z$, qui est de la forme (8) et prendre $s=X$ et

$$
t= \begin{cases}b Y^{2} / 4 & \text { si } b Y^{2} \equiv 0(\bmod 4) \\ M z / 4 & \text { si } M z \equiv 0(\bmod 4) .\end{cases}
$$

La table 1 a été obtenue en prenant $b= \pm p_{1}^{k_{1}}, M=p_{2}^{k_{2}}$ avec pour $i=1,2$, $2 \leq p_{i} \leq 503$ et $p_{1}^{k_{1}} \leq 2^{60}$.

Table 1

\begin{tabular}{ccccc}
\hline$E$ & $s$ & $t$ & $|T(E)|$ & $\sigma$ \\
\hline$E_{2}$ & $1087 \cdot 3187$ & $-2^{29} \cdot 5^{2}$ & 2 & 8.801596 \\
$E_{2}$ & 40103941 & $2^{36} \cdot 7^{2} \cdot 101$ & 2 & 8.485421 \\
$E_{2}$ & $-1693 \cdot 5279$ & $-2^{21} \cdot 691^{2}$ & 2 & 8.294402 \\
$E_{2}$ & $-7 \cdot 173 \cdot 1277 \cdot 2081$ & $-5^{7} \cdot 1217^{2}$ & 2 & 8.273195 \\
$E_{1}$ & 40103941 & $2^{36} \cdot 7^{2} \cdot 101$ & 2 & 8.270467 \\
$E_{2}$ & 118746513373 & $2^{8} \cdot 11^{17}$ & 2 & 8.247556 \\
$E_{1}$ & $-1019801 \cdot 7867$ & $2^{4} \cdot 3^{29} \cdot 11^{4}$ & 2 & 8.034090 \\
$E_{2}$ & $17 \cdot 61 \cdot 239 \cdot 569939$ & $2^{55} \cdot 3^{8} \cdot 5^{2}$ & 2 & 8.005377 \\
$E_{2}$ & $-7 \cdot 17 \cdot 43 \cdot 919903$ & $-3^{2} \cdot 101^{7}$ & 2 & 7.938372 \\
$E_{2}$ & $-3 \cdot 19 \cdot 4861 \cdot 10459$ & $2^{2} \cdot 11^{13} \cdot 137^{2}$ & 2 & 7.909145 \\
\hline
\end{tabular}

En prenant des tordues quadratiques de certaines courbes de la table 1, on obtient la table 2 . 
Table 2

\begin{tabular}{cccccc}
\hline$E$ & $s$ & $t$ & $d$ & $\left|T\left(E^{(d)}\right)\right|$ & $\sigma^{(d)}$ \\
\hline$E_{2}$ & $1087 \cdot 3187$ & $-2^{29} \cdot 5^{2}$ & -3 & 2 & 8.502119 \\
$E_{2}$ & $1087 \cdot 3187$ & $-2^{29} \cdot 5^{2}$ & 5 & 2 & 8.383644 \\
$E_{2}$ & 40103941 & $2^{36} \cdot 7^{2} \cdot 101$ & 5 & 2 & 8.178616 \\
$E_{2}$ & $1087 \cdot 3187$ & $-2^{29} \cdot 5^{2}$ & -15 & 2 & 8.163342 \\
$E_{2}$ & $1087 \cdot 3187$ & $-2^{29} \cdot 5^{2}$ & 17 & 2 & 8.140801 \\
$E_{2}$ & 40103941 & $2^{36} \cdot 7^{2} \cdot 101$ & -7 & 2 & 8.123807 \\
\hline
\end{tabular}

4.2. Supposons maintenant que $x^{2}+A x+8 B=(x-s)(x-t)$. Alors la courbe (12) s'écrit

$$
E_{3}: \quad y^{2}=x(x-s)(x-t),
$$

et a pour discriminant

$$
\Delta_{3}=2^{4} s^{2} t^{2}(s-t)^{2} .
$$

La courbe $E_{3}$ admet $P_{0}, P_{1}=(s, 0)$ et $P_{2}=(t, 0)$ comme points de 2-torsion. En divisant $E_{3}$ par le sous-groupe $\left\{O, P_{0}\right\}$ et en posant $(x, y)=(x+s+t, y)$, on obtient la courbe

$$
E_{4}: \quad y^{2}=x\left(x^{2}+2(s+t) x+(s-t)^{2}\right),
$$

de discriminant

$$
\Delta_{4}=2^{8} s t(s-t)^{4} .
$$

En divisant $E_{3}$ par le sous-groupe $\left\{O, P_{1}\right\}$ et en posant $(x, y)=(x-s+t, y)$, on obtient la courbe

$$
E_{5}: \quad y^{2}=x\left(x^{2}-2(2 s-t) x+t^{2}\right),
$$

de discriminant

$$
\Delta_{5}=2^{8} s t^{4}(s-t) .
$$

En divisant $E_{3}$ par le sous-groupe $\left\{O, P_{2}\right\}$ et en posant $(x, y)=(x+s-t, y)$, on obtient la courbe

$$
E_{6}: \quad y^{2}=x\left(x^{2}+2(s-2 t) x+s^{2}\right),
$$

de discriminant

$$
\Delta_{6}=-2^{8} s^{4} t(s-t) .
$$

Pour les courbes $E_{3}, E_{4}$ et $E_{5}$, on doit trouver des entiers $s$ et $t$, premiers entre eux, pour lesquels le rapport

$$
\frac{\log |s t(s-t)|}{\log \operatorname{rad}(s t(s-t))}
$$

est assez grand, où $\operatorname{rad}(s t(s-t))$ désigne le produit des facteurs premiers de $s t(s-t)$. On peut donc prendre $s$ et $t$ parmi les membres de la relation (8). 
La table 3 a été obtenue à partir des exemples pour la conjecture $a b c$ listés dans [12].

Table 3

\begin{tabular}{ccccc}
\hline$E$ & $s$ & $t$ & $|T(E)|$ & $\sigma$ \\
\hline$E_{4}$ & $2^{30} \cdot 5$ & $-13 \cdot 19^{6}$ & 2 & 8.811944 \\
$E_{4}$ & $2^{10} \cdot 5^{2} \cdot 7^{15}$ & $3^{18} \cdot 23 \cdot 2269$ & 2 & 8.688968 \\
$E_{5}$ & $2^{5} \cdot 3 \cdot 7^{13}$ & $11^{7} \cdot 37^{2} \cdot 353$ & 2 & 8.531805 \\
$E_{5}$ & $2^{5} \cdot 11^{2} \cdot 19^{9}$ & $3^{7} \cdot 7^{11} \cdot 743$ & 2 & 8.482076 \\
$E_{3}$ & $2^{30} \cdot 5$ & $-13 \cdot 19^{6}$ & 4 & 8.461892 \\
$E_{5}$ & $2^{35} \cdot 7^{2} \cdot 17^{2} \cdot 19$ & $5^{15} \cdot 37^{2} \cdot 2311$ & 2 & 8.449373 \\
$E_{5}$ & $2^{8} \cdot 3^{22} \cdot 5^{4}$ & $7 \cdot 29^{2} \cdot 31^{8}$ & 2 & 8.447398 \\
$E_{4}$ & $2^{35} \cdot 7^{2} \cdot 17^{2} \cdot 19$ & $5^{15} \cdot 37^{2} \cdot 2311$ & 2 & 8.438253 \\
$E_{4}$ & $2^{5} \cdot 11^{2} \cdot 19^{9}$ & $3^{7} \cdot 7^{11} \cdot 743$ & 2 & 8.422413 \\
$E_{5}$ & $2^{19} \cdot 13 \cdot 103$ & $3^{11} \cdot 5^{3} \cdot 11^{2}$ & 2 & 8.420203 \\
\hline
\end{tabular}

En prenant des tordues quadratiques convenables des courbes de la table 3 , on obtient la table 4 .

Table 4

\begin{tabular}{lccccc}
\hline$E$ & $s$ & $t$ & $d$ & $T\left(E^{(d)}\right) \mid$ & $\sigma^{(d)}$ \\
\hline$E_{4}$ & $2^{30} \cdot 5$ & $-13 \cdot 19^{6}$ & -3 & 2 & 8.616929 \\
$E_{4}$ & $2^{10} \cdot 5^{2} \cdot 7^{15}$ & $3^{18} \cdot 23 \cdot 2269$ & -3 & 2 & 8.579323 \\
$E_{4}$ & $2^{30} \cdot 5$ & $-13 \cdot 19^{6}$ & 5 & 4 & 8.535178 \\
$E_{4}$ & $2^{10} \cdot 5^{2} \cdot 7^{15}$ & $3^{18} \cdot 23 \cdot 2269$ & 5 & 2 & 8.531330 \\
$E_{4}$ & $2^{10} \cdot 5^{2} \cdot 7^{16}$ & $3^{18} \cdot 7 \cdot 23 \cdot 2269$ & -7 & 2 & 8.500682 \\
$E_{4}$ & $2^{10} \cdot 5^{2} \cdot 7^{15}$ & $3^{18} \cdot 23 \cdot 2269$ & -15 & 2 & 8.433931 \\
\hline
\end{tabular}

5. Points de 3-torsion. Pour avoir $3 P_{0}=O$ sur la courbe (3), il faut avoir $\Psi_{3}(0,0)=0$, et donc $a_{4}^{2}+a_{1} a_{3} a_{4}-a_{3}^{2} a_{2}=0$. Si $a_{3}=0$, alors $a_{4}=0$ et $\Delta=0$. Ainsi $a_{3} \neq 0$ et donc

$$
a_{4}=\frac{1}{2} a_{3}\left(-a_{1} \pm \sqrt{a_{1}^{2}+4 a_{2}}\right)
$$

est entier. On pose $s= \pm \sqrt{a_{1}^{2}+4 a_{2}}$ et $t=a_{3}$. Alors $a_{2}=\left(s^{2}-a_{1}^{2}\right) / 4$ et $a_{4}=t\left(s-a_{1}\right) / 2$. Le changement de variables $(x, y)=\left(x, y+a_{1} x / 2\right)$ donne la courbe d'équation

$$
E_{7}: \quad y^{2}+t y=x^{3}+\frac{1}{4} s^{2} x^{2}+\frac{1}{2} s t x,
$$

de discriminant

$$
\Delta_{7}=t^{3}\left(s^{3}-27 t\right)
$$

En divisant $E_{7}$ par le sous-groupe engendré par $P_{0}$, on trouve la courbe

$$
E_{8}: \quad y^{2}+t y=x^{3}+\frac{1}{4} s^{2} x^{2}-\frac{9}{2} s t x-t\left(s^{3}+7 t\right),
$$


de discriminant

$$
\Delta_{8}=t\left(s^{3}-27 t\right)^{3} .
$$

Pour les courbes $E_{7}$ et $E_{8}$, on peut considérer l'équation $X^{3}-b Y^{3}=M z$, qui est de la forme (8), et prendre $s=X$, et

$$
t= \begin{cases}b Y^{3} / 27 & \text { si } b Y^{3} \equiv 0(\bmod 27) \\ M z / 27 & \text { si } M z \equiv 0(\bmod 27)\end{cases}
$$

Dans la table 5 , on a utilisé $1 \leq b \leq 700, M=p^{k}$ où $p$ est premier vérifiant $2 \leq p \leq 200$ et $p^{k} \leq 2^{60}$.

On peut d'autre part prendre $s=u+4 v$ et $t=u^{2} v$, ce qui donne

$$
\Delta_{7}=u^{6} v^{3}(u+v)(u-8 v)^{2}, \quad \Delta_{8}=u^{2} v(u+v)^{3}(u-8 v)^{6} .
$$

Il suffit ensuite de prendre $u$ et $v$ parmi les membres de l'égalité (8). Dans la table 5, on a utilisé les exemples pour la conjecture $a b c$ listés dans [12]. Les courbes obtenues par cette méthode sont notées $E_{7}^{\prime}$ ou $E_{8}^{\prime}$.

Table 5

\begin{tabular}{ccccc}
\hline$E$ & $s$ & $t$ & $|T(E)|$ & $\sigma$ \\
\hline$E_{8}$ & $811 \cdot 3089$ & $-2^{5} \cdot 41^{8} \cdot 1069$ & 1 & 8.596580 \\
$E_{8}^{\prime}$ & $17 \cdot 457$ & $2^{11} \cdot 3^{4} \cdot 47^{2}$ & 2 & 8.525311 \\
$E_{8}$ & $11 \cdot 47 \cdot 487$ & $3^{3} \cdot 7^{13} \cdot 13$ & 1 & 8.245590 \\
$E_{8}$ & -39367 & $19^{6} \cdot 227$ & 1 & 8.177904 \\
$E_{7}$ & $811 \cdot 3089$ & $-2^{5} \cdot 41^{8} \cdot 1069$ & 3 & 8.157876 \\
$E_{8}$ & 2339 & $3^{20} \cdot 7^{2}$ & 1 & 8.072691 \\
$E_{8}^{\prime}$ & $2^{2} \cdot 4519$ & $2^{28} \cdot 3^{2} \cdot 47$ & 2 & 8.063526 \\
$E_{7}$ & $3 \cdot 811 \cdot 3089$ & $5^{13} \cdot 7^{4} \cdot 199^{3}$ & 3 & 8.048272 \\
$E_{8}^{\prime}$ & $7 \cdot 83$ & $-3^{8} \cdot 5^{4} \cdot 19^{2}$ & 2 & 8.035245 \\
$E_{8}$ & $53 \cdot 809$ & $-3^{4} \cdot 11^{4}$ & 1 & 7.965130 \\
\hline
\end{tabular}

Les tordues quadratiques des courbes de la table 5 donnent la table 6 .

Table 6

\begin{tabular}{cccccc}
\hline$E$ & $s$ & $t$ & $d$ & $\left|T\left(E^{(d)}\right)\right|$ & $\sigma^{(d)}$ \\
\hline$E_{8}$ & $811 \cdot 3089$ & $-2^{5} \cdot 41^{8} \cdot 1069$ & 5 & 1 & 8.405226 \\
$E_{8}$ & $811 \cdot 3089$ & $-2^{5} \cdot 41^{8} \cdot 1069$ & -7 & 1 & 8.368732 \\
$E_{8}^{\prime}$ & $17 \cdot 457$ & $2^{11} \cdot 3^{4} \cdot 47^{2}$ & -3 & 6 & 8.309591 \\
$E_{8}^{\prime}$ & $17 \cdot 457$ & $2^{11} \cdot 3^{4} \cdot 47^{2}$ & 5 & 2 & 8.221360 \\
$E_{8}$ & $811 \cdot 3089$ & $-2^{5} \cdot 41^{8} \cdot 1069$ & -35 & 1 & 8.208450 \\
$E_{8}$ & $811 \cdot 3089$ & $-2^{5} \cdot 41^{8} \cdot 1069$ & 41 & 1 & 8.193856 \\
\hline
\end{tabular}

6. Points de 4-torsion. Pour avoir $4 P_{0}=O$ sur la courbe (4), il faut $\Psi_{4}(0,0)=0$, et donc $A=0$. Les points de 2 -torsion sont alors de la forme 
$P=\left(x,-\left(C x-B C^{2}\right) / 2\right)$, où $x$ vérifie l'équation $\Psi_{2}(x, y)=0$, et donc

$$
(x-B C)\left(4 x^{2}+C^{2} x-C^{3} B\right)=0 .
$$

6.1. Supposons que $4 x^{2}+C^{2} x-C^{3} B$ est irréductible sur $\mathbb{Q}$. On pose alors $s=16 B$ et $t=-C$. L'équation (4) devient donc, après réduction,

$$
E_{9}: \quad y^{2}-4 t x y-4 s t^{2} y=x^{3}+s t x^{2},
$$

et a pour discriminant

$$
\Delta_{9}=-2^{8} s^{4} t^{7}(s-t) .
$$

Le point $2 P_{0}$ est l'unique point de 2 -torsion de $E_{9}$. En divisant $E_{9}$ par le sous-groupe $\left\{O, 2 P_{0}\right\}$, et en simplifiant, on obtient la courbe

$$
E_{10}: \quad y^{2}-2 t x y-\frac{1}{2} s t^{2} y=x^{3}+\frac{1}{4} s t x^{2}-\frac{5}{16} s^{2} t^{2} x+\frac{1}{64} s^{2} t^{3}(3 s-16 t),
$$

de discriminant

$$
\Delta_{10}=2^{4} s^{2} t^{8}(s-t)^{2} .
$$

En divisant $E_{9}$ par le sous-groupe engendré par $P_{0}$, on obtient

$$
E_{11}: \quad y^{2}-2 t x y-\frac{1}{2} s t^{2} y=x^{3}+\frac{1}{4} s t x^{2}+a_{4} x+a_{6},
$$

avec

$$
a_{4}=-\frac{5}{16} s t^{2}(s+16 t), \quad a_{6}=\frac{1}{64} s t^{3}\left(3 s^{2}-192 s t-256 t^{2}\right) .
$$

Le discriminant de $E_{11}$ est

$$
\Delta_{11}=2^{8} s t^{7}(s-t)^{4} .
$$

La courbe $E_{10}$ admet trois points de torsion d'ordre 2 :

$$
\begin{aligned}
& R_{1}=\left(s t / 4, s t^{2} / 2\right), \quad R_{2}=\left(t(s-4 t) / 4, t^{2}(s-2 t) / 2\right), \\
& R_{3}=\left(-3 t s / 4,-s t^{2} / 2\right) .
\end{aligned}
$$

En divisant $E_{10}$ par $\left\{O, R_{1}\right\}$, on trouve $E_{11}$, et en la divisant par $\left\{O, R_{2}\right\}$ on trouve $E_{9}$. Enfin en la divisant par $\left\{O, R_{3}\right\}$, on obtient

$$
E_{12}: \quad y^{2}-2 t x y-\frac{1}{2} s t^{2} y=x^{3}+\frac{1}{4} s t x^{2}+a_{4} x+a_{6},
$$

avec

$$
a_{4}=-\frac{5}{16} s t^{2}(17 s-16 t), \quad a_{6}=\frac{1}{64} s t^{3}\left(275 s^{2}-544 s t+256 t^{2}\right) .
$$

Le discriminant est alors

$$
\Delta_{12}=2^{8} s t^{10}(s-t) .
$$

En utilisant le théorème d'isomorphisme (voir [14]), on a

$$
E_{9} \simeq E_{6}^{(-t)}, \quad E_{10} \simeq E_{3}^{(-t)}, \quad E_{11} \simeq E_{4}^{(-t)}, \quad E_{12} \simeq E_{5}^{(-t)} .
$$

Les grands rapports de Szpiro des courbes $E_{9}, E_{10}, E_{11}$ et $E_{12}$ peuvent donc être obtenus à partir de ceux de $E_{3}, E_{4}, E_{5}$ et $E_{6}$ de la table 3 par des tordues par $-t$. 
6.2. Supposons maintenant que $4 x^{2}+C^{2} x-C^{3} B$ a deux racines rationnelles. On pose alors $C(C+16 B)=s^{2}, C=t$ et donc $B C=\left(s^{2}-t^{2}\right) / 16$. Après réduction, la courbe $(4)$ devient

$$
E_{13}: \quad y^{2}+4 t x y-4 t\left(s^{2}-t^{2}\right) y=x^{3}-\left(s^{2}-t^{2}\right) x^{2},
$$

et admet pour discriminant

$$
\Delta_{13}=2^{8} s^{2} t^{2}(s-t)^{4}(s+t)^{4} .
$$

Les points de 2-torsion de $E_{13}$ sont

$$
P_{1}=2 P_{0}, \quad P_{2}=\left(-2 t(s+t), 2 t(s+t)^{2}\right), \quad P_{3}=\left(2 t(s-t), 2 t(s-t)^{2}\right) .
$$

En divisant $E_{13}$ par le sous-groupe $\left\{O, P_{1}\right\}$ et en réduisant l'équation, on trouve

$$
E_{14}: \quad y^{2}+2 t x y-\frac{1}{2} t\left(s^{2}-t^{2}\right) y=x^{3}-\frac{1}{4}\left(s^{2}-t^{2}\right) x^{2}+a_{4} x+a_{6},
$$

avec

$$
a_{4}=-\frac{5}{16}\left(s^{2}-t^{2}\right)^{2}, \quad a_{6}=-\frac{1}{64}\left(3 s^{2}+13 t^{2}\right)\left(s^{2}-t^{2}\right)^{2} .
$$

Le discriminant est alors

$$
\Delta_{14}=2^{4} s^{4} t^{4}(s-t)^{2}(s+t)^{2} .
$$

En divisant $E_{13}$ par $\left\{O, P_{2}\right\}$, on trouve la courbe

$$
E_{15}: \quad y^{2}+4 t x y-4 t\left(s^{2}-t^{2}\right) y=x^{3}-\left(s^{2}-t^{2}\right) x^{2}+a_{4} x+a_{6},
$$

avec

$$
a_{4}=-20 s t(s+t)^{2}, \quad a_{6}=8 s t(s+t)^{2}\left(2 s^{2}+7 s t-3 t^{2}\right) .
$$

Le discriminant est

$$
\Delta_{15}=2^{10} s t(s+t)^{2}(s-t)^{8} .
$$

Si on divise $E_{13}$ par $\left\{O, P_{3}\right\}$, on retrouve les expressions de $E_{15}$ et $\Delta_{15}$ en remplaçant $t$ par $-t$.

Les points d'ordre 4 de $E_{13}$ sont

$$
\begin{aligned}
& P_{0}, \quad P_{4}=3 P_{0}, \\
& P_{5}=\left(2\left(s^{2}-t^{2}\right),-2(s-t)(s+t)^{2}\right), \\
& P_{6}=\left(2\left(s^{2}-t^{2}\right), 2(s+t)(s-t)^{2}\right) .
\end{aligned}
$$

En divisant $E_{13}$ par le sous-groupe $\left\{O, P_{0}, 2 P_{0}, 3 P_{0}\right\}$ engendré par $P_{0}$, on trouve la courbe

$$
E_{16}: \quad y^{2}+2 t x y-\frac{1}{2} t\left(s^{2}-t^{2}\right) y=x^{3}-\frac{1}{4}\left(s^{2}-t^{2}\right) x^{2}+a_{4} x+a_{6},
$$

avec

$$
\begin{aligned}
& a_{4}=-\frac{5}{16}\left(s^{2}-t^{2}\right)\left(s^{2}-17 t^{2}\right) \\
& a_{6}=-\frac{1}{64}\left(s^{2}-t^{2}\right)\left(3 s^{4}+186 s^{2} t^{2}-445 t^{4}\right) .
\end{aligned}
$$


Le discriminant de $E_{16}$ est

$$
\Delta_{16}=-2^{8} s^{8} t^{2}(s-t)(s+t) .
$$

En divisant $E_{13}$ par le sous-groupe $\left\{O, P_{1}, P_{5}, P_{6}\right\}$, on retrouve $E_{16}$ et $\Delta_{16}$ en permutant $s$ et $t$.

Pour les courbes $E_{13}, E_{14}, E_{15}$ et $E_{16}$, on doit déterminer des entiers $s$ et $t$ pour lesquels le rapport

$$
\frac{\log |s t(s-t)(s+t)|}{\log \operatorname{rad}(s t(s-t)(s+t))}
$$

est assez grand, où $\operatorname{rad}(s t(s-t)(s+t))$ désigne le produit des facteurs premiers de $s t(s-t)(s+t)$. Il suffit alors de les prendre parmi les membres de l'égalité (8).

La table 7 a été obtenue en prenant dans l'équation (8) $n=1,2,1 \leq$ $a \leq|b| \leq 300$ et $M=p^{k}$ où $p$ est un nombre premier vérifiant $2 \leq p \leq 200$ et $p^{k} \leq 2^{30}$.

Table 7

\begin{tabular}{ccccc}
\hline$E$ & $s$ & $t$ & $|T(E)|$ & $\sigma$ \\
\hline$E_{16}$ & $5^{12}$ & $7^{2} \cdot 151 \cdot 181^{2}$ & 2 & 7.965053 \\
$E_{16}$ & $7^{2} \cdot 151 \cdot 181^{2}$ & $5^{12}$ & 2 & 7.963530 \\
$E_{16}$ & $13 \cdot 23^{3}$ & $5^{5} \cdot 7^{2}$ & 2 & 7.952616 \\
$E_{16}$ & $5^{5} \cdot 7^{2}$ & $13 \cdot 23^{3}$ & 2 & 7.941410 \\
$E_{16}$ & $13^{3}$ & $3^{7}$ & 2 & 7.794789 \\
$E_{16}$ & $13^{6} \cdot 103$ & $17^{7}$ & 2 & 7.793004 \\
$E_{16}$ & $3^{7}$ & $13^{3}$ & 2 & 7.792275 \\
$E_{16}$ & $17^{7}$ & $13^{6} \cdot 103$ & 2 & 7.754977 \\
$E_{14}$ & $5^{12}$ & $7^{2} \cdot 151 \cdot 181^{2}$ & 4 & 7.715522 \\
$E_{15}$ & $3^{4} \cdot 10753$ & $2^{23} \cdot 29$ & 4 & 7.691453 \\
\hline \multicolumn{5}{r}{} \\
\hline
\end{tabular}

En prenant des tordues quadratiques de certaines courbes dans la table 7 , on obtient la table 8 .

Table 8

\begin{tabular}{cccccc}
\hline$E$ & $s$ & $t$ & $d$ & $\left|T\left(E^{(d)}\right)\right|$ & $\sigma^{(d)}$ \\
\hline$E_{16}$ & $5^{12}$ & $7^{2} \cdot 151 \cdot 181^{2}$ & -3 & 2 & 7.891402 \\
$E_{16}$ & $7^{2} \cdot 151 \cdot 181^{2}$ & $5^{12}$ & -3 & 2 & 7.889937 \\
$E_{16}$ & $5^{12}$ & $7^{2} \cdot 151 \cdot 181^{2}$ & 5 & 2 & 7.859005 \\
$E_{16}$ & $7^{2} \cdot 151 \cdot 181^{2}$ & $5^{12}$ & 5 & 2 & 7.857564 \\
$E_{16}$ & $5^{12}$ & $7^{2} \cdot 151 \cdot 181^{2}$ & -7 & 2 & 7.838265 \\
$E_{16}$ & $7^{2} \cdot 151 \cdot 181^{2}$ & $5^{12}$ & -7 & 2 & 7.836840 \\
\hline
\end{tabular}

7. Points de 5-torsion. Pour avoir $5 P=0$ sur la courbe (4), il faut $\Psi_{5}(0,0)=0$, et donc $A=B$. On pose $s=C$ et $t=A=B$. L'équation (4) 
donne alors

$$
E_{17}: y^{2}+(s-t) x y-s^{2} t y=x^{3}-s t x^{2},
$$

de discriminant

$$
\Delta_{17}=-s^{5} t^{5}\left(s^{2}+11 s t-t^{2}\right) .
$$

En divisant $E_{17}$ par le groupe engendré par $P_{0}$, on trouve la courbe

$$
E_{18}: \quad y^{2}+(s-t) x y-s^{2} t y=x^{3}-s t x^{2}+a_{4} x+a_{6},
$$

avec

$$
a_{4}=5 s t\left(s^{2}-2 s t-t^{2}\right), \quad a_{6}=s t\left(s^{4}-15 s^{3} t+5 s^{2} t^{2}-10 s t^{3}-t^{4}\right) .
$$

Le discriminant est alors

$$
\Delta_{18}=-s t\left(s^{2}+11 s t-t^{2}\right)^{5} .
$$

Pour les courbes $E_{17}$ et $E_{18}$, on peut prendre $s$ et $t$ parmi les solutions de l'équation (9). La table 9 a été obtenue en prenant $M=p_{1}^{n_{1}} p_{2}^{n_{2}}$, où, pour $i=1,2, p_{i}$ est un nombre premier vérifiant $2 \leq p_{i} \leq 1000$ et $p_{i}^{n_{i}} \leq 2^{40}$.

Table 9

\begin{tabular}{ccccc}
\hline$E$ & $s$ & $t$ & $|T(E)|$ & $\sigma$ \\
\hline$E_{18}$ & $2^{9} \cdot 7^{2} \cdot 163$ & $5^{3} \cdot 11^{3}$ & 1 & 8.243171 \\
$E_{18}$ & $3^{2} \cdot 7^{5} \cdot 11 \cdot 13^{2}$ & -1031 & 1 & 8.074657 \\
$E_{18}$ & $3^{7} \cdot 5^{4} \cdot 1831$ & $2^{10} \cdot 30139$ & 1 & 8.070883 \\
$E_{18}$ & $2^{11} \cdot 5 \cdot 11$ & $-3^{4} \cdot 7$ & 1 & 7.957738 \\
$E_{18}$ & $2^{11} \cdot 3 \cdot 89^{3}$ & -41 & 1 & 7.829967 \\
$E_{18}$ & $3^{12} \cdot 163^{2}$ & $2^{4} \cdot 3435889$ & 1 & 7.827141 \\
$E_{18}$ & $13^{3} \cdot 29$ & $3 \cdot 7 \cdot 47$ & 1 & 7.821050 \\
$E_{18}$ & $5 \cdot 29^{3} \cdot 6529$ & $2^{11} \cdot 7 \cdot 11 \cdot 1103$ & 1 & 7.783123 \\
$E_{17}$ & $2^{9} \cdot 7^{2} \cdot 163$ & $5^{3} \cdot 11^{3}$ & 5 & 7.593892 \\
$E_{17}$ & $3^{7} \cdot 5^{4} \cdot 1831$ & $2^{10} \cdot 30139$ & 5 & 7.500378 \\
\hline
\end{tabular}

En prenant des tordues quadratiques de certaines courbes dans la table 9 , on obtient la table 10 .

Table 10

\begin{tabular}{cccccc}
\hline$E$ & $s$ & $t$ & $d$ & $\left|T\left(E^{(d)}\right)\right|$ & $\sigma^{(d)}$ \\
\hline$E_{18}$ & $2^{9} \cdot 7^{2} \cdot 163$ & $5^{3} \cdot 11^{3}$ & 5 & 1 & 8.090239 \\
$E_{18}$ & $2^{9} \cdot 7^{2} \cdot 163$ & $5^{3} \cdot 11^{3}$ & -7 & 1 & 8.060865 \\
$E_{18}$ & $2^{9} \cdot 7^{2} \cdot 163$ & $5^{3} \cdot 11^{3}$ & -11 & 1 & 8.022682 \\
$E_{18}$ & $3^{7} \cdot 5^{4} \cdot 1831$ & $2^{10} \cdot 30139$ & -3 & 1 & 8.001538 \\
$E_{18}$ & $3^{2} \cdot 7^{5} \cdot 11 \cdot 13^{2}$ & -1031 & -3 & 1 & 7.994573 \\
$E_{18}$ & $3^{7} \cdot 5^{4} \cdot 1831$ & $2^{10} \cdot 30139$ & 5 & 1 & 7.970852 \\
\hline
\end{tabular}


8. Points de 6-torsion. Pour avoir $6 P=0$ sur la courbe (4), il faut $\Psi_{6}(0,0)=0$, et donc que $A, B$ et $C$ vérifient $A \neq B$ et $A^{2}-B C+A C=0$. En éliminant $C$ et en effectuant le changement de variables $(x, y)=\left(A^{2}(A-\right.$ $\left.B)^{2} x, A^{3}(B-A)^{3} y\right)$, la courbe (4) devient

$$
E: \quad y^{2}+(2 A-B) x y+A B(A-B) y=x^{3}+B(A-B) x^{2} .
$$

On a alors

$$
\Psi_{2}^{2}(x, y)=(x+A(A-B))\left(4 x^{2}+B(4 A-3 B) x+A B^{2}(A-B)\right) .
$$

8.1. Supposons que le polynôme $4 x^{2}+B(4 A-3 B) x+A B^{2}(A-B)$ est irréductible sur $\mathbb{Q}$. En posant $s=A$ et $t=B$ la courbe (13) devient

$$
E_{19}: \quad y^{2}+(2 s-t) x y+s t(s-t) y=x^{3}+t(s-t) x^{2},
$$

avec pour discriminant

$$
\Delta_{19}=-s^{2} t^{3}(s-t)^{6}(8 s-9 t) .
$$

Le point $P_{1}=3 P_{0}$ est l'unique point de 2-torsion de $E_{19}$. En divisant $E_{19}$ par le sous groupe $\left\{O, P_{1}\right\}$, on obtient la courbe

$$
E_{20}: \quad y^{2}+(2 s-t) x y+s t(s-t) y=x^{3}+t(s-t) x^{2}+a_{4} x+a_{6},
$$

avec

$$
a_{4}=-5 s(s-t)^{3}, \quad a_{6}=s(s-t)^{3}\left(3 s^{2}-7 s t+3 t^{2}\right) .
$$

Le discriminant est alors

$$
\Delta_{20}=s t^{6}(s-t)^{3}(8 s-9 t)^{2} .
$$

Les points $P_{2}=2 P_{0}$ et $P_{3}=4 P_{0}$ sont des points de torsion d'ordre 3 sur $E_{19}$. En divisant $E_{19}$ par le sous-groupe $\left\{O, P_{2}, P_{3}\right\}$ on obtient la courbe

$$
E_{21}: \quad y^{2}+(2 s-t) x y+s t(s-t) y=x^{3}+t(s-t) x^{2}+a_{4} x+a_{6},
$$

avec

$$
\begin{aligned}
& a_{4}=5 t(s-t)^{2}(2 s-3 t), \\
& a_{6}=t(s-t)^{2}\left(8 s^{3}-33 s^{2} t+43 s t^{2}-19 t^{3}\right) .
\end{aligned}
$$

Le discriminant est alors

$$
\Delta_{21}=-s^{6} t(s-t)^{2}(8 s-9 t)^{3} .
$$

En divisant $E_{19}$ par le sous-groupe engendré par $P_{0}$, on obtient

$$
E_{22}: \quad y^{2}+(2 s-t) x y+s t(s-t) y=x^{3}+t(s-t) x^{2}+a_{4} x+a_{6},
$$

avec

$$
\begin{aligned}
& a_{4}=-5(s-t)\left(s^{3}-2 s^{2} t+5 s t^{2}-3 t^{3}\right), \\
& a_{6}=(s-t)\left(3 s^{5}-13 s^{4} t-24 s^{3} t^{2}+76 s^{2} t^{3}-62 s t^{4}+19 t^{5}\right) .
\end{aligned}
$$

Le discriminant de $E_{22}$ est alors

$$
\Delta_{22}=s^{3} t^{2}(s-t)(8 s-9 t)^{6} .
$$


Pour $s$ et $t$ entiers, on a

$$
\begin{array}{ll}
E_{19}(s, t) \simeq E_{7}\left(2 s-3 t,-t(s-t)^{2}\right), & E_{20}(s, t) \simeq E_{7}\left(4 s-3 t, t^{2}(s-t)\right), \\
E_{21}(s, t) \simeq E_{8}\left(2 s-3 t,-t(s-t)^{2}\right), & E_{22}(s, t) \simeq E_{8}\left(4 s-3 t, t^{2}(s-t)\right) .
\end{array}
$$

Ces relations permettent de déterminer des courbes de la forme $E_{19}, E_{20}$, $E_{21}$ ou $E_{22}$, ayant un grand rapport de Szpiro, à partir des courbes de la table 5 .

8.2. Supposons maintenant que $4 x^{2}+B(4 A-3 B) x+A B^{2}(A-B)$ a deux racines rationnelles. On pose alors $-B(8 A-9 B)=s^{2}$ et $B=t$. En éliminant $A$, et après réduction, la courbe (13) devient

$$
E_{23}: \quad y^{2}-\left(s^{2}-5 t^{2}\right) x y+t^{2}\left(s^{2}-t^{2}\right)\left(s^{2}-9 t^{2}\right) y=x^{3}-2 t^{2}\left(s^{2}-t^{2}\right) x^{2},
$$

et a pour discriminant

$$
\Delta_{23}=s^{2} t^{6}(s-t)^{6}(s+t)^{6}(s+3 t)^{2}(s-3 t)^{2} .
$$

Les trois points de 2 -torsion de $E_{23}$ sont $P_{1}=3 P_{0}, P_{2}$ et $P_{3}$ donnés par

$$
\begin{aligned}
& P_{2}=\left(t^{2}(s+t)(s-3 t),-t^{3}(s+t)^{2}(s-3 t)\right), \\
& P_{3}=\left(t^{2}(s-t)(s+3 t), t^{3}(s-t)^{2}(s+3 t)\right) .
\end{aligned}
$$

En divisant $E_{23}$ par le sous-groupe $\left\{O, P_{1}\right\}$, on trouve

$$
\begin{aligned}
E_{24}: \quad y^{2}-\frac{1}{2}\left(s^{2}-5 t^{2}\right) x y+\frac{1}{8} t^{2}( & \left.s^{2}-t^{2}\right)\left(s^{2}-9 t^{2}\right) y \\
& =x^{3}-\frac{1}{2} t^{2}\left(s^{2}-t^{2}\right) x^{2}+a_{4} x+a_{6},
\end{aligned}
$$

avec

$$
\begin{aligned}
& a_{4}=-\frac{5}{256}\left(s^{2}-t^{2}\right)^{3}\left(s^{2}-9 t^{2}\right), \\
& a_{6}=\frac{1}{4096}\left(s^{2}-t^{2}\right)^{3}\left(s^{2}-9 t^{2}\right)\left(3 s^{4}+2 s^{2} t^{2}-69 t^{4}\right) .
\end{aligned}
$$

Le discriminant est alors

$$
\Delta_{24}=s^{4} t^{12}(s-t)^{3}(s+t)^{3}(s+3 t)(s-3 t) .
$$

En divisant $E_{23}$ par le sous-groupe $\left\{O, P_{2}\right\}$, on trouve

$$
\begin{aligned}
E_{25}: \quad y^{2}-\left(s^{2}-5 t^{2}\right) x y+t^{2}\left(s^{2}-t^{2}\right) & \left(s^{2}-9 t^{2}\right) y \\
& =x^{3}-2 t^{2}\left(s^{2}-t^{2}\right) x^{2}+a_{4} x+a_{6},
\end{aligned}
$$

avec

$$
\begin{aligned}
& a_{4}=5 s t^{3}(s+t)^{3}(s-3 t), \\
& a_{6}=s t^{3}(s+t)^{3}(s-3 t)\left(s^{4}-11 s^{2} t^{2}-14 s t^{3}+12 t^{4}\right) .
\end{aligned}
$$

Le discriminant est alors

$$
\Delta_{25}=-s t^{3}(s-t)^{12}(s+t)^{3}(s+3 t)^{4}(s-3 t) .
$$

En divisant $E_{23}$ par le sous-groupe $\left\{O, P_{3}\right\}$, on retrouve $E_{25}$ et $\Delta_{25}$ en remplaçant $t$ par $-t$. 
Les points de 3 -torsion de $E_{23}$ sont $P_{4}=2 P_{0}$ et $P_{5}=4 P_{0}=-P_{4}$. En divisant $E_{23}$ par le sous-groupe $\left\{O, P_{4}, P_{5}\right\}$, on trouve

$E_{26}: y^{2}-\left(s^{2}-5 t^{2}\right) x y+t^{2}\left(s^{2}-t^{2}\right)\left(s^{2}-9 t^{2}\right) y=x^{3}-2 t^{2}\left(s^{2}-t^{2}\right) x^{2}+a_{4} x+a_{6}$,

avec

$$
\begin{aligned}
& a_{4}=-5 t^{2}\left(s^{2}-t^{2}\right)^{2}\left(s^{2}+3 t^{2}\right), \\
& a_{6}=-t^{2}\left(s^{2}-t^{2}\right)^{2}\left(s^{6}+6 s^{4} t^{2}-7 s^{2} t^{4}+64 t^{6}\right) .
\end{aligned}
$$

Le discriminant est alors

$$
\Delta_{26}=s^{6} t^{2}(s-t)^{2}(s+t)^{2}(s+3 t)^{6}(s-3 t)^{6} .
$$

En divisant $E_{23}$ par le sous-groupe engendré par $P_{0}$, on trouve

$$
\begin{aligned}
& E_{27}: \quad y^{2}-\frac{1}{2}\left(s^{2}-5 t^{2}\right) x y+\frac{1}{8} t^{2}\left(s^{2}-t^{2}\right)\left(s^{2}-9 t^{2}\right) y \\
&=x^{3}-\frac{1}{2} t^{2}\left(s^{2}-t^{2}\right) x^{2}+a_{4} x+a_{6},
\end{aligned}
$$

avec

$$
\begin{aligned}
& a_{4}=-\frac{5}{256}\left(s^{2}-t^{2}\right)\left(s^{6}-11 s^{4} t^{2}+275 s^{2} t^{4}-777 t^{6}\right), \\
& a_{6}=\frac{1}{4096}\left(s^{2}-t^{2}\right)\left(3 s^{10}-31 s^{8} t^{2}-2850 s^{6} t^{4}+31234 s^{4} t^{6}\right. \\
& \left.-131633 s^{2} t^{8}+136045 t^{10}\right) .
\end{aligned}
$$

Le discriminant est alors

$$
\Delta_{27}=s^{12} t^{4}(s-t)(s+t)(s+3 t)^{3}(s-3 t)^{3} .
$$

En divisant $E_{23}$ par le sous-groupe engendré par $2 P_{0}$ et $P_{2}$, on trouve

$$
\begin{aligned}
E_{28}: \quad y^{2}-\left(s^{2}-5 t^{2}\right) x y+t^{2}\left(s^{2}-t^{2}\right) & \left(s^{2}-9 t^{2}\right) y \\
& =x^{3}-2 t^{2}\left(s^{2}-t^{2}\right) x^{2}+a_{4} x+a_{6},
\end{aligned}
$$

avec

$$
\begin{array}{r}
a_{4}=5 t(s+t)\left(s^{6}-10 s^{5} t+28 s^{4} t^{2}-29 s^{3} t^{3}+2 s^{2} t^{4}+3 s t^{5}-3 t^{6}\right), \\
a_{6}=t(s+t)\left(s^{10}-24 s^{9} t+157 s^{8} t^{2}-437 s^{7} t^{3}+462 s^{6} t^{4}-50 s^{5} t^{5}\right. \\
\left.-67 s^{4} t^{6}-17 s^{3} t^{7}+71 s^{2} t^{8}+64 s t^{9}-64 t^{10}\right) .
\end{array}
$$

Le discriminant est alors

$$
\Delta_{28}=-s^{3} t(s-t)^{4}(s+t)(s+3 t)^{12}(s-3 t)^{3} .
$$

En divisant $E_{23}$ par le sous-groupe engendré par $2 P_{0}$ et $P_{3}$, on retrouve $E_{28}$ et $\Delta_{28}$ en remplaçant $t$ par $-t$.

Pour les courbes $E_{23}$ à $E_{28}$, on peut prendre $s$ et $t$ parmi les membres de (8). La table 11 a été obtenue en prenant $n=1,1 \leq a \leq|b| \leq 300$ et $M=p^{k}$ avec $p$ premier, vérifiant $2 \leq p \leq 300$ et $p^{k} \leq 2^{32}$. 
Table 11

\begin{tabular}{ccccc}
\hline$E$ & $s$ & $t$ & $|T(E)|$ & $\sigma$ \\
\hline$E_{28}$ & 7 & $-3^{5}$ & 2 & 7.806270 \\
$E_{28}$ & $7^{6}$ & -37 & 2 & 7.624470 \\
$E_{25}$ & 7 & $-3^{5}$ & 1 & 7.569888 \\
$E_{28}$ & $3 \cdot 59$ & $-2^{3} \cdot 23$ & 2 & 7.466010 \\
$E_{28}$ & $7^{2}$ & $2^{8} \cdot 19$ & 2 & 7.431017 \\
$E_{28}$ & $-7^{2}$ & $2^{8} \cdot 19$ & 2 & 7.431017 \\
$E_{28}$ & $19 \cdot 101^{2}$ & $2^{6} \cdot 557$ & 2 & 7.415144 \\
$E_{26}$ & $7^{6}$ & -37 & 4 & 7.384042 \\
$E_{28}$ & $7^{6}$ & 37 & 2 & 7.376711 \\
$E_{25}$ & $2^{8} \cdot 3 \cdot 19$ & $7^{2}$ & 6 & 7.376579 \\
\hline
\end{tabular}

En prenant des tordues quadratiques de certaines courbes dans la table 11 , on obtient la table 12 .

Table 12

\begin{tabular}{cccccc}
\hline$E$ & $s$ & $t$ & $d$ & $\left|T\left(E^{(d)}\right)\right|$ & $\sigma^{(d)}$ \\
\hline$E_{28}$ & 7 & $-3^{5}$ & -3 & 6 & 7.686752 \\
$E_{28}$ & 7 & $-3^{5}$ & 5 & 2 & 7.636405 \\
$E_{28}$ & 7 & $-3^{5}$ & -7 & 2 & 7.604852 \\
$E_{28}$ & $7^{6}$ & -37 & -3 & 6 & 7.572905 \\
$E_{28}$ & $7^{6}$ & -37 & 5 & 2 & 7.550027 \\
$E_{28}$ & 7 & $-3^{5}$ & -15 & 2 & 7.537694 \\
\hline
\end{tabular}

9. Points de 7-torsion. Si $7 P_{0}=O$ sur la courbe $(4)$, alors $\Psi_{7}(0,0)=0$, et donc $A, B$ et $C$ vérifient $A^{3}=B C(B-A)$. Ainsi $(A / C, B / C)$ est un point rationnel sur la courbe singulière $Y^{2}-X Y=X^{3}$. En posant $Y=X s / t$ avec $s$ et $t$ entiers, on trouve $X=s(s-t) / t^{2}, Y=s^{2}(s-t) / t^{3}$ et on peut donc prendre $A=s t(s-t), B=s^{2}(s-t)$ et $C=t^{3}$. Alors (4) devient

$$
E_{29}: \quad y^{2}-\left(s^{2}-s t-t^{2}\right) x y-s^{2} t^{3}(s-t) y=x^{3}-s^{2} t(s-t) x^{2},
$$

et a pour discriminant

$$
\Delta_{29}=s^{7} t^{7}(s-t)^{7}\left(s^{3}-8 s^{2} t+5 s t^{2}+t^{3}\right) .
$$

En divisant $E_{29}$ par le sous-groupe engendré par $P_{0}$, on obtient

$E_{30}: y^{2}-\left(s^{2}-s t-t^{2}\right) x y-s^{2} t^{3}(s-t) y=x^{3}-s^{2} t(s-t) x^{2}+a_{4} x+a_{6}$,

avec

$$
\begin{aligned}
& a_{4}=-5 s t(s-t)\left(s^{2}-s t+t^{2}\right)\left(s^{3}+2 s^{2} t-5 s t^{2}+t^{3}\right) \\
& \begin{array}{r}
a_{6}=-s t(s-t)\left(s^{9}+9 s^{8} t\right. \\
+37 s^{7} t^{2}+70 s^{6} t^{3}-132 s^{5} t^{4} \\
+ \\
\left.+211 s^{4} t^{5}-182 s^{3} t^{6}+76 s^{2} t^{7}-18 s t^{8}+t^{9}\right) .
\end{array}
\end{aligned}
$$


Le discriminant est alors

$$
\Delta_{30}=s t(s-t)\left(s^{3}-8 s^{2} t+5 s t^{2}+t^{3}\right)^{7} .
$$

On peut prendre alors $s$ et $t$ parmi les membres de (8) ou parmi les solutions de (10).

La table 13 a été obtenue en utilisant les bons exemples pour la conjecture $a b c[12]$ et en prenant dans l'équation (10) $M=p^{k}$, où $p$ est premier vérifiant $2 \leq p \leq 600$ et $p^{k} \leq 2^{40}$.

Table 13

\begin{tabular}{ccccc}
\hline$E$ & $s$ & $t$ & $|T(E)|$ & $\sigma$ \\
\hline$E_{30}$ & 11 & 9 & 1 & 8.757316 \\
$E_{30}$ & $2 \cdot 3^{5}$ & -1 & 1 & 7.444604 \\
$E_{29}$ & $2^{15} \cdot 13$ & $31^{2} \cdot 59$ & 7 & 7.362457 \\
$E_{30}$ & $2^{4} \cdot 19$ & 283 & 1 & 7.327803 \\
$E_{30}$ & $2^{4} \cdot 139^{2}$ & $5^{2} \cdot 23^{3}$ & 1 & 7.308972 \\
$E_{30}$ & $53^{3}$ & $2^{4} \cdot 3^{2} \cdot 13 \cdot 79$ & 1 & 7.231768 \\
$E_{30}$ & $5^{4}$ & $2^{5} \cdot 17$ & 1 & 7.205254 \\
$E_{30}$ & $17^{2} \cdot 229$ & $29^{2}$ & 1 & 7.169130 \\
$E_{30}$ & $2^{15} \cdot 13$ & $31^{2} \cdot 59$ & 1 & 7.138013 \\
$E_{30}$ & 11423 & $3^{3} \cdot 349$ & 1 & 7.114253 \\
\hline
\end{tabular}

En prenant des tordues quadratiques des courbes de la table 13, on obtient la table 14 .

Table 14

\begin{tabular}{cccccc}
\hline$E$ & $s$ & $t$ & $d$ & $\left|T\left(E^{(d)}\right)\right|$ & $\sigma^{(d)}$ \\
\hline$E_{30}$ & 11 & 9 & -3 & 1 & 8.371586 \\
$E_{30}$ & 11 & 9 & -11 & 1 & 8.034917 \\
$E_{30}$ & 11 & 9 & 13 & 1 & 7.998441 \\
$E_{30}$ & 11 & 9 & 33 & 1 & 7.816835 \\
$E_{30}$ & 11 & 9 & -39 & 1 & 7.787702 \\
$E_{30}$ & 11 & 9 & -1 & 1 & 7.637494 \\
\hline
\end{tabular}

10. Points de 8-torsion. Pour avoir $8 P_{0}=O$ sur la courbe (4), il faut $\Psi_{8}(0,0)=0$, et donc $A, B$ et $C$ vérifient $A^{2} B-A^{2} C+3 A B C-2 B^{2} C=0$. Alors $(A / C, B / C)$ est une solution de l'équation $X^{2} Y-X^{2}+3 X Y-2 Y^{2}=0$. On pose $Y=X s / t$ avec $s$ et $t$ entiers. On obtient

$$
X=\frac{(2 s-t)(s-t)}{s t}, \quad Y=\frac{(2 s-t)(s-t)}{t^{2}},
$$

ce qui permet de prendre $A=t(2 s-t)(s-t), B=s(2 s-t)(s-t)$ et $C=s t^{2}$. 
Après réduction, l'équation (4) devient

$E_{31}: \quad y^{2}-\left(2 s^{2}-4 s t+t^{2}\right) x y-s^{3} t(s-t)(2 s-t) y=x^{3}-s^{2}(s-t)(2 s-t) x^{2}$, et a pour discriminant

$$
\Delta_{31}=s^{8} t^{2}(s-t)^{8}(2 s-t)^{4}\left(8 s^{2}-8 s t+t^{2}\right) .
$$

On ne considère dans cette partie que le cas général où $\Psi_{2}(x, y)$ n'admet pas obligatoirement trois racines rationnelles. En divisant $E_{31}$ par le sous-groupe $\left\{O, 4 P_{0}\right\}$, on obtient

$$
\begin{aligned}
E_{32}: \quad y^{2}-\left(2 s^{2}-4 s t+t^{2}\right) & x y-s^{3} t(s-t)(2 s-t) y \\
& =x^{3}-s^{2}(s-t)(2 s-t) x^{2}+a_{4} x+a_{6},
\end{aligned}
$$

avec

$$
\begin{aligned}
& a_{4}=-5 s^{4}(s-t)^{4}, \\
& a_{6}=-s^{4}(s-t)^{4}\left(3 s^{4}-11 s^{3} t+16 s^{2} t^{2}-8 s t^{3}+t^{4}\right) .
\end{aligned}
$$

Le discriminant est alors

$$
\Delta_{32}=s^{4} t^{4}(s-t)^{4}(2 s-t)^{8}\left(8 s^{2}-8 s t+t^{2}\right)^{2} .
$$

En divisant $E_{31}$ par le sous-groupe engendré par $2 P_{0}$, on obtient

$$
\begin{aligned}
E_{33}: \quad y^{2}-\left(2 s^{2}-4 s t+t^{2}\right) & x y-s^{3} t(s-t)(2 s-t) y \\
& =x^{3}-s^{2}(s-t)(2 s-t) x^{2}+a_{4} x+a_{6},
\end{aligned}
$$

avec

$$
\begin{aligned}
& a_{4}=-5 s^{2}(s-t)^{2}\left(17 s^{4}-34 s^{3} t+25 s^{2} t^{2}-8 s t^{3}+t^{4}\right) \\
& a_{6}=-s^{2}(s-t)^{2}\left(275 s^{8}-1185 s^{7} t+2177 s^{6} t^{2}-2211 s^{5} t^{3}\right. \\
& \left.\quad+1354 s^{4} t^{4}-513 s^{3} t^{5}+119 s^{2} t^{6}-16 s t^{7}+t^{8}\right) .
\end{aligned}
$$

Le discriminant est alors

$$
\Delta_{33}=s^{2} t^{8}(s-t)^{2}(2 s-t)^{4}\left(8 s^{2}-8 s t+t^{2}\right)^{4} .
$$

En divisant $E_{31}$ par le sous-groupe engendré par $P_{0}$, on obtient

$$
\begin{aligned}
E_{34}: \quad y^{2}-\left(2 s^{2}-4 s t+t^{2}\right) & x y-s^{3} t(s-t)(2 s-t) y \\
& =x^{3}-s^{2}(s-t)(2 s-t) x^{2}+a_{4} x+a_{6},
\end{aligned}
$$

avec

$$
\begin{array}{r}
a_{4}=-5 s(s-t)\left(17 s^{6}-51 s^{5} t+59 s^{4} t^{2}-33 s^{3} t^{3}+5 s^{2} t^{4}+3 s t^{5}-t^{6}\right), \\
a_{6}=-s(s-t)\left(275 s^{10}-1460 s^{9} t+3362 s^{8} t^{2}-4388 s^{7} t^{3}+3721 s^{6} t^{4}\right. \\
\left.-2315 s^{5} t^{5}+1123 s^{4} t^{6}-392 s^{3} t^{7}+77 s^{2} t^{8}-3 s t^{9}-t^{10}\right) .
\end{array}
$$

Le discriminant est

$$
\Delta_{34}=s t^{4}(s-t)(2 s-t)^{2}\left(8 s^{2}-8 s t+t^{2}\right)^{8} .
$$


La courbe $E_{32}$ admet 3 points de 2-torsion :

$$
\begin{aligned}
R_{1}= & \left(s^{2}(s-t)(3 s-2 t), s^{2}(s-t)^{3}(3 s-t)\right), \\
R_{2}= & \left(-\frac{1}{4}\left(4 s^{4}-12 s^{3} t+16 s^{2} t^{2}-8 s t^{3}+t^{4}\right),\right. \\
\left.\quad-\frac{1}{8}\left(8 s^{6}-48 s^{5} t+96 s^{4} t^{2}-96 s^{3} t^{3}+50 s^{2} t^{4}-12 s t^{5}+t^{6}\right)\right), & \left(-s^{2}(s-t)(s-2 t),-s^{2}(s-t)^{2}\left(s^{2}-4 s t+t^{2}\right)\right) .
\end{aligned}
$$

En divisant $E_{32}$ par $\left\{O, R_{1}\right\}$ on retrouve $E_{33}$ et en la divisant par $\left\{O, R_{2}\right\}$ on retrouve $E_{31}$. Enfin en divisant $E_{32}$ par $\left\{O, R_{3}\right\}$, on trouve

$$
\begin{aligned}
E_{35}: \quad y^{2}-\left(2 s^{2}-4 s t+t^{2}\right) x y & -s^{3} t(s-t)(2 s-t) y \\
& =x^{3}-s^{2}(s-t)(2 s-t) x^{2}+a_{4} x+a_{6},
\end{aligned}
$$

avec

$$
\begin{aligned}
& a_{4}=-5 s^{2}(s-t)^{2}\left(s^{4}-2 s^{3} t-7 s^{2} t^{2}+8 s t^{3}-t^{4}\right) \\
& \begin{array}{r}
a_{6}=-s^{2}(s-t)^{2}\left(3 s^{8}-17 s^{7} t+129 s^{6} t^{2}-275 s^{5} t^{3}+164 s^{4} t^{4}\right. \\
+ \\
\left.+53 s^{3} t^{5}-73 s^{2} t^{6}+16 s t^{7}-t^{8}\right) .
\end{array}
\end{aligned}
$$

Le discriminant est alors

$$
\Delta_{35}=-s^{2} t^{2}(s-t)^{2}(2 s-t)^{16}\left(8 s^{2}-8 s t+t^{2}\right) .
$$

On peut procéder de la même façon en utilisant les trois points de 2-torsion de $E_{33}$. Mais seul le point

$$
\begin{aligned}
R_{4}=\left(s(s-t)\left(11 s^{2}-10 s t+2 t^{2}\right),\right. & \left.s(s-t)\left(11 s^{4}-31 s^{3} t+27 s^{2} t^{2}-9 s t^{3}+t^{4}\right)\right),
\end{aligned}
$$

sert à déterminer une nouvelle courbe elliptique. En divisant $E_{33}$ par $\left\{O, R_{4}\right\}$, on obtient

$$
\begin{aligned}
E_{36}: \quad y^{2}-\left(2 s^{2}-4 s t+t^{2}\right) & x y-s^{3} t(s-t)(2 s-t) y \\
& =x^{3}-s^{2}(s-t)(2 s-t) x^{2}+a_{4} x+a_{6},
\end{aligned}
$$

avec

$$
\begin{aligned}
a_{4}= & -5 s(s-t)\left(273 s^{6}-819 s^{5} t+955 s^{4} t^{2}\right. \\
& \left.-545 s^{3} t^{3}+157 s^{2} t^{4}-21 s t^{5}+t^{6}\right), \\
a_{6}= & -s(s-t)\left(18963 s^{10}-96180 s^{9} t+210338 s^{8} t^{2}-259492 s^{7} t^{3}\right. \\
& +198433 s^{6} t^{4}-97355 s^{5} t^{5}+30685 s^{4} t^{6} \\
& \left.-6054 s^{3} t^{7}+705 s^{2} t^{8}-43 s t^{9}+t^{10}\right) .
\end{aligned}
$$

Le discriminant est alors

$$
\Delta_{36}=s t^{16}(s-t)(2 s-t)^{2}\left(8 s^{2}-8 s t+t^{2}\right)^{2} .
$$


Comme les expressions $s-t, 2 s-t$ et $8 s^{2}-8 s t+t^{2}$ apparaissent dans les discriminants $\Delta_{31}, \ldots, \Delta_{36}$, on peut prendre $s$ et $t$ parmi les membres de (8) ou parmi les solutions de (11).

La table 15 a été obtenue en utilisant les exemples de [12] d'une part, et en prenant dans l'équation (11) $M=p^{k}$, où $p$ est premier vérifiant $2 \leq p \leq$ 1000 et $p^{k} \leq 2^{30}$.

\section{Table 15}

\begin{tabular}{ccccc}
\hline$E$ & $s$ & $t$ & $|T(E)|$ & $\sigma$ \\
\hline$E_{34}$ & $2^{3}$ & -22619 & 2 & 7.431643 \\
$E_{36}$ & $2^{3}$ & -22619 & 2 & 7.431101 \\
$E_{35}$ & $31^{2}$ & $3^{8}$ & 4 & 7.416867 \\
$E_{36}$ & $2^{10}$ & $3^{5} \cdot 11$ & 2 & 7.397627 \\
$E_{34}$ & $2^{10}$ & $3^{5} \cdot 11$ & 2 & 7.369815 \\
$E_{36}$ & $2^{11} \cdot 3$ & $113^{2}$ & 2 & 7.359048 \\
$E_{34}$ & $2^{11} \cdot 3$ & $113^{2}$ & 2 & 7.358475 \\
$E_{36}$ & $2^{8}$ & $3 \cdot 23^{2}$ & 2 & 7.354622 \\
$E_{36}$ & $31^{2}$ & $3^{8}$ & 2 & 7.342342 \\
$E_{34}$ & 311 & $-2 \cdot 5^{5}$ & 2 & 7.288486 \\
\hline
\end{tabular}

En prenant des tordues quadratiques des courbes de la table 15, on obtient la table 16 .

Table 16

\begin{tabular}{cccccc}
\hline$E$ & $s$ & $t$ & $d$ & $T\left(E^{(d)}\right) \mid$ & $\sigma^{(d)}$ \\
\hline$E_{34}$ & $2^{3}$ & -22619 & -3 & 2 & 7.383118 \\
$E_{36}$ & $2^{3}$ & -22619 & -3 & 2 & 7.382594 \\
$E_{34}$ & $2^{3}$ & -22619 & 5 & 2 & 7.361658 \\
$E_{36}$ & $2^{3}$ & -22619 & 5 & 2 & 7.361143 \\
$E_{35}$ & $31^{2}$ & $3^{8}$ & -3 & 2 & 7.359289 \\
$E_{36}$ & $2^{10}$ & $3^{5} \cdot 11$ & -3 & 2 & 7.338752 \\
\hline
\end{tabular}

11. Autres cas. Une étude similaire pour les courbes elliptiques ayant un point de torsion $m \in\{9,10,12\}$ n'a pas donné de résultats probants pour le rapport de Szpiro, et ne sera donc pas développée. Ceci est dû au grand nombre de facteurs sans grands exposants dans l'expression du discriminant. A titre d'exemple, soit

$$
E_{0}: y^{2}+a_{1} x y+a_{3} y=x^{3}+a_{2} x^{2},
$$

avec

$$
\begin{aligned}
& a_{1}=-\left(s^{4}+2 s^{3} t+2 s^{2} t^{2}+2 s t^{3}-t^{4}\right), \\
& a_{2}=-s t^{2}(s+t)\left(s^{2}+t^{2}\right)\left(s^{2}+s t+t^{2}\right), \\
& a_{3}=s t^{5}(s+t)(s-t)\left(s^{2}+t^{2}\right)\left(s^{2}+s t+t^{2}\right) .
\end{aligned}
$$


Alors $P_{0}=(0,0)$ est un point de torsion d'ordre 12 sur $E_{0}$, mais le discriminant

$$
\Delta_{0}=s^{12} t^{12}(s+t)^{6}(s-t)^{2}\left(t^{2}+s^{2}\right)^{3}\left(s^{2}+s t+t^{2}\right)^{4}\left(s^{2}+4 s t+t^{2}\right),
$$

admet plusieurs facteurs, ce qui augmente les contraintes dans la recherche d'un rapport de Szpiro assez grand.

\section{References}

[1] M. Ayad, Points S-entiers des courbes elliptiques, Manuscripta Math. 76 (1992), 305-324.

[2] C. Batut, D. Bernardi, H. Cohen and M. Olivier, PARI-GP, a computer system for number theory, Version 1.39, ftp://megrez.ceremab.u-bordeaux.fr/ $\mathrm{pub} /$ pari/.

[3] H. Cohen, A Course in Computational Algebraic Number Theory, Grad. Texts in Math. 138, Springer, Berlin, 1993.

[4] I. Connell, APECS, Version 3.7, 1996, ftp://math.mcgill.ca/pub/apecs/.

[5] J. Cremona, Algorithms for Modular Elliptic Curves, Cambridge University Press, Cambridge, 1992.

[6] E. Fouvry, M. Nair et G. Tenenbaum, L'ensemble exceptionnel dans la conjecture de Szpiro, Bull. Soc. Math. France 120 (1992), 485-506.

[7] M. Hindry and J. H. Silverman, The canonical height and integral points on elliptic curves, Invent. Math. 93 (1988), 419-450.

[8] D. Husemoller, Elliptic Curves, Grad. Texts in Math. 111, Springer, Berlin, 1986.

[9] D. S. Kubert, Universal bounds on the torsion of elliptic curves, Proc. London Math. Soc. 33 (1976), 193-237.

[10] T. Nagell, Recherches sur l'arithmétique des cubiques planes du premier genre dans un domaine de rationalité quelconque, Nova Acta Soc. Sci. Upsal, Ser. IV 15, 6 (1952), 1-66.

[11] A. Nitaj, Algorithms for finding good examples for the abc and Szpiro conjectures, Experiment. Math. 3 (1993), 223-230.

[12] - Tables of good abc-examples, preprint, Saarbrücken, 1997.

[13] J. Oesterlé, Nouvelles approches du "théorème" de Fermat, Séminaire Bourbaki 1987-88, no. 694, Astérisque 161-162 (1988), 165-186.

[14] J. H. Silverman, The Arithmetic of Elliptic Curves, Grad. Texts in Math. 106, Springer, Berlin, 1986.

[15] SIMATH, a computer algebra system, Version 3.10.3, Simath-Gruppe, Saarbrücken, 1996, ftp://ftp.math.uni-sb.de:/pub/simath.

[16] L. Szpiro, Propriétés numériques du faisceau dualisant relatif, dans : Pinceaux de Courbes de Genre au Moins Deux, Astérisque 86 (1981), 44-78.

[17] —, Discriminant et conducteur, dans : Séminaire sur les Pinceaux de Courbes Elliptiques, Astérisque 183 (1990), 7-17.

[18] H. M. Tschöpe and H. G. Zimmer, Computation of the Néron-Tate height on elliptic curves, Math. Comp. 48 (1987), 351-370. 
[19] J. Vélu, Isogénies entre courbes elliptiques, C. R. Acad. Sci. Paris Sér. I Math. 273 (1971), 238-241.

[20] B. M. M. de Weger, $A+B=C$ and big $I I ' s$, Quart. J. Math. Oxford Ser. (2) 49 (1998), 105-128.

Fb 9, Mathematik

Universität des Saarlandes

Postfach 151150

D-66041 Saarbrücken, Germany

E-mail: nitaj@math.uni-sb.de 